\title{
Revised Nomenclature for Fatty Liver Disease: Cutting through the Confusion
}

\author{
Khalid Alsawat ${ }^{1}$, Almoutaz Hashim ${ }^{2}$, Mohamed Alboraie ${ }^{3}$ and Yasser Fouad*4 \\ ${ }^{1}$ Liver Research Center, Department of Medicine, College of Medicine, King Saud University, Riyadh, Kingdom of Saudi Arabia; \\ ${ }^{2}$ Department of Medicine, University of Jeddah, Jeddah, Kingdom of Saudi Arabia; ${ }^{3}$ Department of Internal Medicine, Al-Azhar \\ University, Cairo, Egypt; ${ }^{4}$ Department of Gastroenterology and Endemic Medicine, Minia University, Minia, Egypt
}

Citation of this article: Alsawat $\mathrm{K}$, Hashim A, Alboraie M, Fouad Y. Revised nomenclature for fatty liver disease: Cutting through the confusion. J Clin Transl Hepatol 2020;8(3):354355. doi: $10.14218 /$ JCTH.2020.00049.

Nomenclature of disease is pivotal for both clinicians and patients, as well as for other stakeholders, and serves as a critical nosological reference point. The term 'non-alcoholic fatty liver disease (NAFLD)' was introduced to a broader audience by Ludwig and colleagues in $1980 .^{1}$ The authors coined this term, trying to describe a clinical entity from fatty liver disease that is not caused by increased alcohol intake. In their description and subsequent guidelines, it was pointed out that diagnosis is based on the exclusion of significant alcohol intake and other liver diseases.

With the rapidly evolving rise in the disease's prevalence and advancement of our understanding of its pathomechanisms and the identification of risk genes, there is growing need for initiatives aimed at increasing allocation of funding and resources, raising public awareness, and promoting social care to promote medical care. However, it has been become more obvious that the current nomenclature and definition of the disease are significant barriers that hinder achieving these goals. ${ }^{2}$ Thus, it is currently more obvious that the adopted nomenclature "NAFLD" is unclear, as nonspecific implies "anti-definition" and leads to imprecise communication between different stakeholders, with the potential for causing patient harm. As such, it possibly results in limitation of awareness of the disease, increasing related stigma, and decreasing the allocation of health care and funding resources. ${ }^{3}$

Besides the dichotomization of fatty liver into alcoholic and nonalcoholic fatty liver in the current definition, there is no universally accepted cut-off of low daily alcohol intake that can distinguish between the entities and there is lack of an accurate enough instrument to detect small differences in alcohol intake. In addition, this simple dichotomization does not take in account the negative consequences of alcohol intake, which even at low-level are shaped by a multitude of factors including genetic, dietary and impact of the gut microbiota. ${ }^{4}$ Recent evidence suggests that some gut bacteria can produce alcohol contributing to liver damage. ${ }^{5}$

Abbreviations: MAFLD, metabolic-associated fatty liver disease; NAFLD, nonalcoholic fatty liver disease; NASH, nonalcoholic steatohepatitis.

Received: 28 May 2020; Revised: 4 August 2020; Accepted: 27 August 2020

*Correspondence to: Yasser Fouad, Gastroenterology and Hepatology, Endemic Medicine Department, Minia University, Main Road, Minia 11432, Egypt. Tel: +201091318555, E-mail: yasserfouad10@yahoo.com
Furthermore, with this advancement of knowledge, we are realizing more and more that, similar to other complex diseases, the spectrum of the disease displays huge heterogeneity, with likely multiple sub-phenotypes having differential clinical presentation, outcomes, and therapeutic response. ${ }^{6}$ Adopting a clear and consistent terminology is critical in any context to try to deconvolute this heterogeneity and pave the way for personalization of medicine. Hence, there is a real need to revisit the nomenclature and definition systems of diseases and to update them according to new findings to reflect the current understanding of the disease appropriately.

To tackle these challenges, a collaboration of an international consortium of 32 experts from 22 countries undertook a detailed analysis of the accuracy of fatty liver disease nomenclature, definition, and disease spectrum, including sources of heterogeneity. This consortium proposed metabolic dysfunction-associated fatty liver disease (MAFLD) as more appropriate terminology for this disease, and they developed a simplified and yet comprehensive "positive" criteria for diagnosis of the disease. ${ }^{6,7}$ The new nomenclature aims to shift the disease diagnosis towards a diagnosis of inclusion, based on the presence of metabolic dysfunction, the crucial driver of the disease development and progression, rather than the current definition which is solely based on the exclusion. The new criteria are based on incorporation of "positive criteria", regardless of alcohol consumption or other concomitant liver diseases. This new definition helps to identify a homogenous group of patients and could ultimately guide efforts for stratification of patients with MAFLD and informing "innovative clinical trials and precision of medicine".

This proposal represents a paradigm shift in the field, as it not only overcomes the significant limitations of the current "anti-definition" but it also ensures that the diagnosis of MAFLD is clear and well defined. The new criteria is for identification of steatosis with one of the different modalities (imaging, blood biomarker, or histology), in addition to one of three criteria, namely overweight or obesity, type 2 diabetes mellitus, or evidence of metabolic abnormalities. ${ }^{7}$ The proposed changes to the definition of fatty liver disease will have ramifications for patients, researchers, and clinicians. They have the potential to lead to more succinct, evidencedriven clinical management algorithms and may help to ensure standardization of care. With various compounds in clinical trials, these changes have the premise that recruited cohorts will be more homogeneous, and these are expected to accelerate the development of effective therapy.

Another intriguing aspect of this novel proposal for redefining fatty liver disease is the concept that MAFLD should be 
considered as the umbrella terminology that is used to describe the continuum spectrum of the disease. This spectrum should extend to include patients with cirrhosis with vanished features of steatosis, who used to be termed as having "cryptogenic cirrhosis". In this scope, this disease severity would be assessed by the degree of inflammation and fibrosis, akin to other liver diseases, abandoning by this the simple and misleading dichotomous classification into nonalcoholic steatohepatitis "NASH" vs. "non-NASH".

This proposed redefinition could have significant implications, including improving discovering noninvasive predictor scores for the disease and the performance of the ongoing clinical trials. Notably, while NASH is currently one of the endpoints of clinical trials, it has been shown that it is usually harder to be met. ${ }^{8,9}$ Future studies are urgently required to explore the impact of the implementation of this new proposal.

In conclusion, the redefinition of metabolic fatty liver disease will likely be a crucial step in a long path towards improving the management of these patients. Further deliberate efforts are required to capitalize on this landmark initiative to maximize efforts in combating the growing burden of the disease.

\section{Funding}

None to declare.

\section{Conflict of interest}

The authors have no conflict of interest related to this publication.

\section{Author contributions}

Conceptualization and writing of the manuscript (KA, $A H$, $M A, Y F)$.

\section{References}

[1] Ludwig J, Viggiano TR, McGill DB, Oh BJ. Nonalcoholic steatohepatitis: Mayo Clinic experiences with a hitherto unnamed disease. Mayo Clin Proc 1980;55: 434-438.

[2] Fouad Y, Waked I, Bollipo S, Gomaa A, Ajlouni Y, Attia D. What's in a name? Renaming 'NAFLD' to 'MAFLD'. Liver Int 2020;40:1254-1261. doi: 10. 1111/liv. 14478.

[3] Manns MP, Burra P, Sargent J, Horton R, Karlsen TH. The Lancet-EASL Commission on liver diseases in Europe: overcoming unmet needs, stigma, and inequities. Lancet 2018;392:621-622. doi: 10.1016/S0140-6736(18) 31734-3.

[4] Eslam M, Sanyal AJ, George J. Toward more accurate nomenclature for fatty liver diseases. Gastroenterology 2019;157:590-593. doi: 10.1053/j.gastro. 2019.05.064.

[5] Yuan J, Chen C, Cui J, Lu J, Yan C, Wei X, et al. Fatty liver disease caused by high-alcohol-producing klebsiella pneumoniae. Cell Metab 2019;30:1172. doi: 10.1016/j.cmet.2019.11.006.

[6] Eslam M, Sanyal AJ, George J. MAFLD: A consensus-driven proposed nomenclature for metabolic associated fatty liver disease. Gastroenterology 2020; 158:1999-2014.e1. doi: 10.1053/j.gastro.2019.11.312.

[7] Eslam M, Newsome PN, Sarin SK, Anstee QM, Targher G, Romero-Gomez M, et al. A new definition for metabolic dysfunction-associated fatty liver disease: An international expert consensus statement. J Hepatol 2020;73:202-209. doi: 10.1016/j.jhep.2020.03.039.

[8] Eslam M, Alvani R, Shiha G. Obeticholic acid: towards first approval for NASH. Lancet 2019;394:2131-2133. doi: 10.1016/S0140-6736(19)32963-0.

[9] Younossi ZM, Ratziu V, Loomba R, Rinella M, Anstee QM, Goodman Z, et al. Obeticholic acid for the treatment of non-alcoholic steatohepatitis: interim analysis from a multicentre, randomised, placebo-controlled phase 3 trial. Lancet 2019;394:2184-2196. doi: 10.1016/S0140-6736(19)33041-7. 